\title{
On Linial's Conjecture for Split Digraphs
}

\author{
Maycon Sambinelli ${ }^{1 *}$ Cândida Nunes da Silva ${ }^{2}$, Orlando Lee ${ }^{1 \dagger}$ \\ ${ }^{1}$ Institute of Computing - University of Campinas (Unicamp) \\ 13083-852 - Campinas - SP - Brazil \\ ${ }^{2}$ Department of Computing - Federal University of São Carlos (Ufscar) \\ 18052-780 - Sorocaba - SP - Brazil \\ \{msambinelli,lee\}@ic.unicamp.br, candida@ufscar.br
}

\begin{abstract}
In this paper we show that Linial's Conjecture holds for two classes of split digraphs, namely the spider digraphs and the $k$-loose digraphs.
\end{abstract}

\section{Introduction}

The digraphs considered in this text do not contain loops or parallel arcs and by path we mean directed path. Let $D$ be a digraph. We denote by $V(P)$ the set of vertices of a path $P$. The size of a path $P$, denoted by $|P|$, is $|V(P)|^{1}$. We denote by $\lambda(D)$ the size of the longest path in $D$ and by $\alpha(D)$ the size of a maximum stable set. A path partition $\mathcal{P}$ of $D$ is a set of vertex-disjoint paths of $D$ that cover $V(P)$. We say that $\mathcal{P}$ is an optimal path partition if there is no path partition $\mathcal{P}^{\prime}$ of $D$ such that $\left|\mathcal{P}^{\prime}\right|<|\mathcal{P}|$. We denote by $\pi(D)$ the size of an optimal path partition of a digraph $D$.

Dilworth [Dilworth 1950] showed that for every transitive acyclic digraph $D$ we have $\pi(D)=\alpha(D)$. Note that this equality is not valid for any digraph; for example, if $D$ is a directed cycle with 5 vertices, then $\pi(D)=1$ and $\alpha(D)=2$. However, Gallai and Milgram [Gallai and Milgram 1960] have shown that $\pi(D) \leq \alpha(D)$ for every digraph $D$.

Greene and Kleitman [Greene and Kleitman 1976] proved a generalization of Dilworth's Theorem described next. Let $k$ be a positive integer. The $k$-norm of a path partition $\mathcal{P}$, denoted by $|\mathcal{P}|_{k}$, is defined as $|\mathcal{P}|_{k}=\sum_{P \in \mathcal{P}} \min \{|P|, k\}$. We say that $\mathcal{P}$ is a $\boldsymbol{k}$-optimal path partition if there is no path partition $\mathcal{P}^{\prime}$ such that $\left|\mathcal{P}^{\prime}\right|_{k}<|\mathcal{P}|_{k}$. We denote by $\pi_{k}(D)$ the $k$-norm of a $k$-optimal path partition of $D$. A $\boldsymbol{k}$-partial coloring $\mathcal{C}^{k}$ is a set of $k$ disjoint stable sets called color classes (empty color classes are allowed). The weight of a $k$-partial coloring $\mathcal{C}^{k}$, denoted by $\left\|\mathcal{C}^{k}\right\|$, is defined as $\left\|\mathcal{C}^{k}\right\|=\sum_{C \in \mathcal{C}^{k}}|C|$. We say that $\mathcal{C}^{k}$ is an optimal $\boldsymbol{k}$-partial coloring if there is no $k$-partial coloring $\mathcal{B}^{k}$ such that $\left\|\mathcal{B}^{k}\right\|>\left\|\mathcal{C}^{k}\right\|$. We denote by $\alpha_{k}(D)$ the weight of an optimal $k$-partial coloring of $D$. Given these definitions, what Greene and Kleitman [Greene and Kleitman 1976] showed was that for every transitive acyclic digraph $D$, we have $\pi_{k}(D)=\alpha_{k}(D)$. Note that $\pi(D)=\pi_{1}(D)$ and $\alpha(D)=\alpha_{1}(D)$. Thus, Dilworth's Theorem is a particular case of Greene-Kleitman's Theorem in which $k=1$.

As Gallai-Milgram's Theorem extends Dilworth's Theorem, it is a natural question whether Greene-Kleitman's Theorem can be extended to digraphs in general. More precisely, is it true that for every digraph $D$ we have that $\pi_{k}(D) \leq \alpha_{k}(D)$ ? Linial [Linial 1981] conjectured that the answer for this question is positive.

\footnotetext{
* Supported by National Counsel of Technological and Scientific Development - CNPq (grant 141216/2016-6).

$\dagger$ Supported by National Counsel of Technological and Scientific Development - CNPq (grants 311373/2015-1 and 477692/2012-5).

${ }^{1}$ Usually $|P|$ denotes the length of a path (number of arcs), but here it denotes the number of vertices.
} 
Linial's Conjecture [Linial 1981]. Let $D$ be a digraph and $k$ be a positive integer. Then, $\pi_{k}(D) \leq \alpha_{k}(D)$.

Linial's Conjecture remains open, but we know it holds for acyclic digraphs [Saks 1979], bipartite digraphs [Berge 1982], digraphs which contain a Hamiltonian path [Berge 1982], $k=1$ [Linial 1978], $k=2$ [Berger and Hartman 2008] and $k \geq \lambda(D)-3$ [Herskovics 2013]. In this paper we give partial results on Linial's Conjecture for split digraphs.

\section{Split digraphs}

Let $D$ be a digraph and let $X \subseteq V(D)$. We denote by $D[X]$ the subdigraph of $D$ induced by $X$. A digraph $D$ is a split digraph if there is a partition of $V(D)$ into two sets $X$ and $Y$, such that $D[X]$ is a tournament and $D[Y]$ is a stable set. We shall use the notation $D[X, Y]$ to indicate that $D$ is a split digraph with such partition $\{X, Y\}$.

In this section we shall prove an approximation to Linial's Conjecture for split digraphs, i. e., that $\pi_{k}(D) \leq \alpha_{k}(D)+1$ for every split digraph $D$, as stated in Theorem 1 . For that, we need Rédei's Theorem and Lemmas 1 and 2 below.

Rédei’s Theorem [Rédei 1934]. Every tournament contains a Hamiltonian path.

Lemma 1. Let $D[X, Y]$ be a split digraph. Then, $\pi_{k}(D) \leq|Y|+\min \{|X|, k\}$.

Proof. By Rédei's Theorem, the tournament $D[X]$ contains a path $P$ such that $V(P)=$ $X$. Let $\mathcal{P}=\{P\} \cup\{(y): y \in Y\}$. Clearly, $\mathcal{P}$ is a path partition of $D$ for which $|\mathcal{P}|_{k}=\min \{|X|, k\}+|Y|$. Therefore, $\pi_{k}(D) \leq|\mathcal{P}|_{k}=\min \{|X|, k\}+|Y|$.

Lemma 2. Let $D[X, Y]$ be a split digraph. Then, $\alpha_{k}(D) \geq|Y|+\min \{|X|, k-1\}$. Moreover, when $|X|<k$, we have that $\alpha_{k}(D)=|V(D)|$.

Proof. First, suppose that $|X| \leq k-1$. Let $\mathcal{C}^{k}=\{Y\} \cup\{\{x\}: x \in X\}$. Note that $\mathcal{C}^{k}$ is a $k$-partial coloring of $D$ with $\left\|\mathcal{C}^{k}\right\|=|V(D)|$. Therefore, $\alpha_{k}(D)=\left\|\mathcal{C}^{k}\right\|=$ $|Y|+|X|=|Y|+\min \{|X|, k-1\}$ and the result follows. We may assume that $|X| \geq k$. Let $\mathcal{C}^{k}=\{Y\} \cup\{\{x\}: x \in S\}$, where $S \subseteq X$ such that $|S|=k-1$. Clearly, $\mathcal{C}^{k}$ is a $k$-partial coloring for which $\left\|\mathcal{C}^{k}\right\|=|Y|+k-1$. Therefore, $\alpha_{k}(D) \geq\left\|\mathcal{C}^{k}\right\|=$ $|Y|+k-1=|Y|+\min \{|X|, k-1\}$.

Theorem 1. Let $D[X, Y]$ be a split digraph. Then, $\pi_{k}(D) \leq \alpha_{k}(D)+1$.

Proof. The result follows immediately from Lemmas 1 and 2.

In Section 2.1 we introduce $k$-loose digraphs and show that Linial's Conjecture holds for them and in Section 2.2 we show that it holds for spider digraphs [Hoàng 1985].

\section{1. $k$-loose digraphs}

A split digraph $D[X, Y]$ is $\boldsymbol{k}$-loose if either $|X|<k$ or there is a $S \subseteq X$ such that $|S|=k$ and no vertex $y \in Y$ is adjacent to every vertex in $S$. A split digraph $D[X, Y]$ that is not $k$-loose is called $\boldsymbol{k}$-tight. We show in this section that Linial's Conjecture holds for every $k$-loose digraph (Theorem 2) and for split digraphs such that $|X| \leq k$ (Theorem 3). For that, we need Lemmas 3 and 4 below.

Lemma 3. Let $D[X, Y]$ be a split digraph. Then, $D$ is $k$-loose if and only if $\alpha_{k}(D) \geq$ $|Y|+\min \{|X|, k\}$. 
Proof. Consider that $D$ is $k$-loose. If $|X|<k$, then by Lemma 2, $\alpha_{k}(D)=|V(D)|=$ $|Y|+|X| \geq|Y|+\min \{|X|, k\}$. We may thus assume that $|X| \geq k$ and there is $S \subseteq X$ such that $|S|=k$ and no vertex $y \in Y$ is adjacent to every vertex in $S$. Assume $S=$ $\left\{x_{1}, x_{2}, \ldots, x_{k}\right\}$ and let $\mathcal{C}_{0}^{k}=\left\{C_{1}, C_{2}, \ldots, C_{k}\right\}$ be a $k$-partial coloring where $C_{i}=\left\{x_{i}\right\}$ for $i=1,2, \ldots, k$. For each $y \in Y$ choose some vertex $x_{i}$ not adjacent to $y$ (which exists by definition) and add $y$ in color class $C_{i}$. The $k$-partial coloring $\mathcal{C}^{k}$ thus obtained has weight $|Y|+k=|Y|+\min \{|X|, k\}$ as expected.

Conversely, consider that $\alpha_{k}(D) \geq|Y|+\min \{|X|, k\}$. If $|X|<k$, then $D$ is $k$ loose by definition. So, we may assume that $|X| \geq k$ and, whence, $\alpha_{k}(D) \geq|Y|+k$. We conclude that $\mathcal{C}^{k}$ must have exactly $k$ vertices of $X$, besides all $|Y|$ vertices from $Y$. Let $S=\left\{x: x \in C_{i} \cap X\right.$ for $\left.i=1,2, \ldots, k\right\}$. Since all vertices of $Y$ belong to $\mathcal{C}^{k}$, then there is no vertex in $Y$ which is adjacent to every vertex of $S$. Therefore, $D$ is $k$-loose.

Theorem 2. Let $D[X, Y]$ be a $k$-loose split digraph. Then, $\pi_{k}(D) \leq \alpha_{k}(D)$.

Proof. By Lemma 3, $\alpha_{k}(D) \geq|Y|+\min \{|X|, k\}$. On the other hand, by Lemma 1 $\pi_{k}(D) \leq|Y|+\min \{|X|, k\}$ and the result follows.

Lemma 4. Let $D[X, Y]$ be a split digraph such that $\lambda(D)>|X|$. Then, $\pi_{k}(D) \leq \alpha_{k}(D)$. Proof. If $\alpha_{k}(D)=|V(D)|$, then the result follows trivially. Thus, we may assume that $\alpha_{k}(D)<|V(D)|$. By Lemma 2 we have that $|X| \geq k$ and also that $\alpha_{k}(D) \geq$ $|Y|+\min \{|X|, k-1\}=|Y|+k-1$. Since $\lambda(D)>|X|$, there exists a path $P$ in $D$ such that $|P|=|X|+1$. Let $\mathcal{P}=\{P\} \cup\{(v): v \notin V(P)\}$. Clearly, $\mathcal{P}$ is a path partition of $D$ and $|\mathcal{P}|_{k}=|Y|+k-1$. Therefore, $\pi_{k}(D) \leq|\mathcal{P}|_{k} \leq \alpha_{k}(D)$.

Theorem 3. Let $D[X, Y]$ be a split digraph such that $|X| \leq k$. Then, $\pi_{k}(D) \leq \alpha_{k}(D)$. Proof. If $D$ is $k$-loose, then the result follows by Theorem 2. So, we may assume that $D$ is not $k$-loose. Hence, $|X|=k$ and there exists a vertex $y \in Y$ which is adjacent to every vertex of $X$. Therefore, $D[X \cup\{y\}]$ is a tournament and by Rédei's Theorem it has a Hamiltonian path $P$ such that $|P|=|X|+1$. As $P$ is a path in $D$ as well, we conclude that $\lambda(D) \geq|X|+1$ and the result follows by Lemma 4 .

\subsection{Spider digraphs}

We denote by $\mathcal{N}(v)$ the set of vertices that are adjacent to $v \in V(D)$ (regardless the direction of the arcs). A split digraph $D[X, Y]$ is spider [Hoàng 1985] if $(i)$ $|X|=|Y| \geq 2$; and $(i i)$ there exists a bijective function $f: X \rightarrow Y$ such that either $\mathcal{N}(x)=\{f(x)\}$ for all $x \in X$ (in this case, we say that $D$ is a thin spider) or $\mathcal{N}(x)=Y-f(x)$ for all $x \in X$ (in this case, we say that $D$ is a thick spider). Note that thin spider digraphs are $k$-loose, but thick spider digraphs are $k$-tight, as long as $|X|>k$. The following theorem shows that Linial's Conjecture holds for spider digraphs.

Theorem 4. Let $D[X, Y]$ be a spider digraph. Then, $\pi_{k}(D) \leq \alpha_{k}(D)$.

Proof. Let $\ell=|X|=|Y|$. If $\ell \leq k$, then the result follows by Theorem 3. Thus, we may assume that $|X|>k$. Clearly, $\pi_{k}(D) \leq|V(D)|$ and we deduce that $\alpha_{k}<|V(D)|$. If $D$ is a thin spider digraph, whence $k$-loose, the result follows by Theorem 2 . Therefore, we may assume that $D$ is a thick spider graph. Since $D[X]$ is a tournament, by Rédei's Theorem, there exists a path $P$ such that $V(P)=X$. Let $P=\left(x_{1}, x_{2}, \ldots, x_{\ell}\right)$. Since $D$ is a thick spider digraph, there exists one single vertex $y_{i} \in Y$ that is not adjacent to $x_{i}$, for $i=1, \ldots, \ell$. Note that if $\lambda(D)>|X|$, then the result follows by Lemma 4. So we may assume that $\lambda(D) \leq|X|$. 
Let $P x_{i}$ denote the subpath $\left(x_{1}, x_{2}, \ldots, x_{i}\right)$ and let $x_{i} P$ denote the subpath $\left(x_{i}, x_{i+1}, \ldots, x_{\ell}\right)$. We denote by $W \circ Q$ the concatenation of two paths $W$ and $Q$.

Claim 1: If $x_{i} \in X, y_{j} \in Y$ and $i<j$, then $\left(x_{i}, y_{j}\right) \in A(D)$.

We prove this claim by induction on $i$. If $i=1$, assume by contradiction that $\left(y_{j}, x_{1}\right) \in$ $A(D)$; then $P^{\prime}=\left(y_{j}, x_{1}\right) \circ P$ is a path in $D$ such that $\left|P^{\prime}\right|=|X|+1$, a contradiction. Hence, $\left(x_{1}, y_{j}\right) \in A(D)$. Consider now $i>1$. Recall that $y_{j}$ is adjacent to every vertex in $X-\left\{x_{j}\right\}$. Thus, $y_{j}$ is adjacent to every vertex of $V\left(P x_{i}\right)$. By induction hypothesis, we have $\left(x_{i-1}, y_{j}\right) \in A(D)$. Suppose by contradiction that $\left(y_{j}, x_{i}\right) \in A(D)$. Then, there is a path $P^{\prime}=P x_{i-1} \circ\left(x_{i-1}, y_{j}, x_{i}\right) \circ x_{i} P$ such that $\left|P^{\prime}\right|=|X|+1$, a contradiction. Therefore, $\left(x_{i}, y_{j}\right) \in A(D)$. This completes the proof of Claim 1 .

Claim 2: If $x_{i} \in X, y_{j} \in Y$ and $j<i$, then $\left(y_{j}, x_{i}\right) \in A(D)$.

We omit the proof of Claim 2, as it is analogous to that of Claim 1.

We claim that both $P_{0}=\left(x_{1}, y_{2}, x_{3}, y_{4}, \ldots\right)$ and $P_{1}=\left(y_{1}, x_{2}, y_{3}, x_{4}, \ldots\right)$ are paths in $D$. By Claim 1 we have that $\left(x_{i}, y_{i+1}\right) \in A(D)$ for $i=1,3, \ldots$, and by Claim 2 we have that $\left(y_{j}, x_{j+1}\right) \in A(D)$ for $j=2,4, \ldots$. Hence $P_{0}$ is a path in $D$. The proof is analogous for $P_{1}$. Clearly, $\mathcal{P}=\left\{P_{0}, P_{1}\right\}$ is a path partition of $D$. Moreover, $\left|P_{0}\right|=\left|P_{1}\right|=\ell$ and $|\mathcal{P}|_{k}=2 \min \{\ell, k\}=2 k$. Since $|X|>k$, we have that $\min \{\ell, k\}=$ $k \leq|X|-1=|Y|-1$. Thus, $|\mathcal{P}|_{k}=2 k \leq k+|Y|-1$. On the other hand, by Lemma 2, $\alpha_{k}(D) \geq|Y|+\min \{|X|, k-1\}=|Y|+k-1$. Therefore, $\pi_{k}(D) \leq|\mathcal{P}|_{k}=2 k \leq$ $|Y|+k-1 \leq \alpha_{k}(D)$.

\section{Conclusion}

We showed that Linial's Conjecture holds for $k$-loose digraphs and for some subclasses of $k$-tight digraphs, namely those with $|X|=k$ and the thick spider digraphs. It is easy to see that for $k$-tight digraphs, $\alpha_{k}(D)=|Y|+k-1$. Therefore, it is clear that any approach to prove Linial's Conjecture for $k$-tight digraphs must involve finding a path partition with $k$-norm less than or equal to $|Y|+k-1$. We are currently working on this idea.

\section{References}

Berge, C. (1982). k-optimal partitions of a directed graph. European Journal of Combinatorics, 3(2):97-101.

Berger, E. and Hartman, I. B.-A. (2008). Proof of Berge's strong path partition conjecture for $k=2$. European Journal of Combinatorics, 29(1):179-192.

Dilworth, R. P. (1950). A decomposition theorem for partially ordered sets. Annals of Mathematics, 51(1):161-166.

Gallai, T. and Milgram, A. N. (1960). Verallgemeinerung eines graphentheoretischen satzes von rédei. Acta Sci Math, 21:181-186.

Greene, C. and Kleitman, D. J. (1976). The structure of Sperner $k$-families. Journal of Combinatorial Theory, Series A, 20(1):41-68

Herskovics, D. (2013). Proof of Berge's path partition conjecture for $k \geq \lambda-3$. Technical Report TR-2013-08, Egerváry Research Group, Budapest.

Hoàng, C. T. (1985). Perfect Graphs. PhD thesis, School of Computer Science, McGill University Montreal.

Linial, N. (1978). Covering digraphs by paths. Discrete Mathematics, 23(3):257-272.

Linial, N. (1981). Extending the Greene-Kleitman theorem to directed graphs. Journal of Combinatorial Theory, Series A, 30(3):331-334.

Rédei, L. (1934). Ein kombinatorischer satz. Acta Litt. Szeged, 7(39-43):97.

Saks, M. (1979). A short proof of the existence of $k$-saturated partitions of partially ordered sets. Advances in Mathematics, 33(3):207-211. 